\title{
A Comparative Study of Type 1 Singleton Fuzzy Logic Systems in Machining Application
}

\author{
Pascual Noradino Montes Dorantes ${ }^{1}$, Marco Aurelio Jiménez Gómez ${ }^{1}$, \\ Xavier Cantú Rodriguez ${ }^{2}$, and Gerardo Maximiliano Méndez ${ }^{3}$ \\ ${ }^{1}$ Departamento de Ingeniería Industrial, División de estudios de posgrado e investigación, \\ Instituto Tecnológico de Ciudad Victoria, Tamaulipas, Mexico \\ ${ }^{2}$ Universidad del Valle de México (Campus Monterrey), Nuevo León, Mexico \\ ${ }^{3}$ Posgrado en Ingeniería Mecatrónica Instituto Tecnológico de Nuevo León, \\ Nuevo León, Mexico \\ pascualresearch@gmail.com
}

\begin{abstract}
This paper presents a comparison of two different techniques of Soft Computing (SC), in union with a novel approach of a hybrid model with Central Composite Design (CCD) with capacity of performing a rule reduction, without loss of reliability, and also performs a reduction of the computational time spent in prediction. Results obtained with this method show better performance thought generating a minimum error rate when it is compared with the Adaptive Network Fuzzy Inference Systems (ANFIS), or with the least squares method in case of pure the Fuzzy Singleton system.
\end{abstract}

Keywords: Fuzzy logic, hybrid model, ANFIS, CCD, T1SFLS.

\section{Introduction}

Dynamics of modern manufacturing processes require systems that can be fast, reliable and safe for products and persons that operating them. The science and technology developed in XX century provide some tools in the form of intelligent systems. The Soft Computing (SC) has proven to be reliable in industrial applications, but modern systems need to deal with a lot of information provided by multiple sources. Also, they need to allocate the resources in a safe, reliable and different manner [1]. There are many real word examples in which some data is missing. In these cases the mathematical models such as Least Squares (LS) are useful in obtaining an output response for the function that represents the systems. This prediction and the historical data serve as inputs for the mathematical model that serves as a database in modeling the expert system. These classes of new systems must be able to adapt in fulfillment of the requirements, but these needs, new models capable to manage: knowledge and reasoning such as humans do. Fuzzy Inference Systems (FIS) can satisfy these requirements and can be modeled in a combination with other SC techniques to create or simplify these models. e.g., the combination of neural networks (NN) with Fuzzy Logic Systems (FLS) creates the ANFIS model. 
The aim of this paper is test the SC techniques of fuzzy systems in their version singleton in the form of pure Type 1 Singleton fuzzy logic system (T1SFLS) and hybrid systems (ANFIS) and evaluate their performances to find a model capable to work on a real time and to provide a simplification of the system itself. This is made with the use of design techniques such as CCD, and serves to reduce the complexity of the design and the implementation of these intelligent systems. These techniques were used for tuning the parameters (in this case for a machining process).

The organization of this paper is as follows: Section 1 already reviewed above, Section 2 shows the state of art. Section 3 presents a general overview of the SC techniques (FLS \& ANFIS). Section 4 presents the proposed method. Section 5 presents the results obtained for a machining process. Section 6 presents conclusions and finally. Section 7 presents some references.

\section{State of the Art}

The machining process is defined as a high precision method to model pieces for both people and industry. The most ancient machining process was made 150,000 years ago in the form of carved stone [2]. Basically, the machining processes are classified into three forms: turning, grinding and milling in the other hand drilling is added by [3]. These processes are used to give the shape of the pieces. Drilling is a critical operation because it provides the necessary holes in the assembly process [4]. The use of advanced techniques in manufacturing processes such as SC provides the chance to simulate the performance of the system without expends resources, also can be evaluated different states of the system under different conditions

In [5] is mentioned that the machining in some materials is a difficult task because there are no actual specification manuals in literature. Also, technical specifications of materials do not determine a specific kind of tool or parameters to make the process. These ideas were proposed by the International Journal of Automation Technology (IJAT) in their call for papers of a special number Machining of difficult to cut materials in 2013.

The performance of machining processes can be simulated with FIS or FLS as is mentioned in [6] also this method can be used as a control system. One of the advantages of this technique is its fast response and fast adaptation without overshoot. [7] shows the complications for drilling composites and advanced materials. They say that these processes need new approaches or new methods and technologies to adapt and tuning the parameters to drill these kinds of materials.

The ANFIS model developed by Jang in 1993 [8], now they are talking, which this is one of the forms of hybridization of the FL (Fuzzy Logic) and NN and says "ANFIS is a combination of the best part of fuzzy logic and neural networks" as mentioned in [9] and they makes a review of ANFIS models for machining process. In [10] is proposed a combination of FLS with Design of Experiments (DOE) in CCD form with good results, but their proposal uses individual base inference.

[11] shows in its proposal the use of response surface methodology, but they does not present the use of SC techniques. Their technique has a restriction of three variables when the model is multivariate, and in fact, with more than three variables 
the additional variables need to be fixed, and this condition finally produces an exponential grow of the model.

[12] presents an optimized model based on the CCD / FLS and CCD/ANFIS with the approach of composed base inference. This approach reduces the complexity of the model and the rule base. In this mentioned model one of the advantages is that all states of the system are covered by the combinatorial itself. In this combinatory some parameters are fixed, and they evaluate the behavior of the remaining parameters. Another advantage or these models is that they do not need a hard mathematical model to develop FLS or ANFIS (e.g. individual base inference). The requirements for these applications are based only on historical data, and then are modeled and tuning to obtain a prediction.

Other approaches proposed in literature such as: Naïve Bayes and Ant Colony Optimization for combinatorial optimization [13], in [14] show the use of evolutionary strategies to obtain a model called evolutionary design of experiments. In [15], they used multiple techniques of SC to adjust an extrusion blow molding process; this uses a Taguchi design method, full factorial design and fractional factorial design to create a hybrid combination.

\section{Preliminaries}

\subsection{Soft Computing Techniques Based on Fuzzy Logic (FL)}

\section{Fuzzy Logic}

The FL was born in 1965 with the propositions made by Zadeh, these ones create a function in the form (1) as a matrix of rules that evaluate in quantitative form linguistic labels with a membership degree, without limitations of Boolean logic such as the human reasoning. The first proposal [16] includes crisp values. Later, an analysis presented in 1975 [17] includes linguistics in this technique. This theory becomes reality in 1975 with the Mamdani controller that is described in its paper [18].

$$
\text { Rule } n: \text { IF } X_{1} \text { is } A_{1} \text { and ... and } X i \text { is Ai, Then } Y \text { is } B
$$

\section{ANFIS}

In 1993 the ANFIS model appears in literature as an effort to enhance pure soft computing models with the hybrid union of FL and NN. This model is capable to adapt and learn from feedback. In the actual ANFIS applications the basic model proposed by Jang [19] does not have evolved and continues with the same proposition. The novel approaches found in literature deals in the field of learning and training with the use of: Back propagation [20], least squares [21], morphological transforms [22], and decision trees [23].

The ANFIS system is equivalent to Sugeno system (see Figure 1), and the model is a 5 layer NN (See Figure 5c). The basis for this NN is actually a fuzzy system. The main difference with FLS and ANFIS systems is the prediction algorithm, this is 
based on several transformations ruled in the assignation of weights to the inputs, and the main advantage is they do not need a defuzzifier at all [19].

In an ANFIS system every layer is dedicated to perform a specific function (mathematical operation), and these layers are composed of neurons that only can perform simply mathematical calculus due their simplicity. The use of this model is that it can simplify the complex problems of mathematical model, but it still needs the formulation of the fuzzy rules to assemble the ANFIS model.

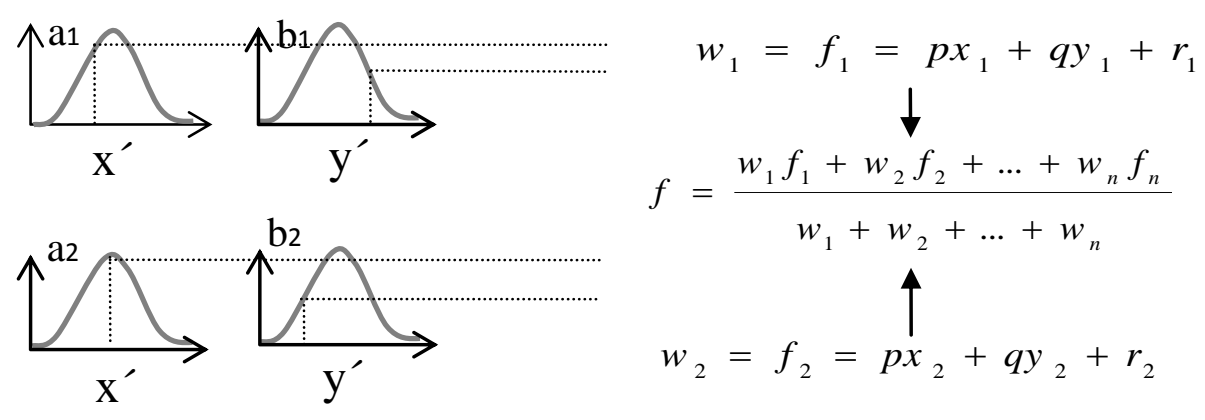

Fig. 1. Sugeno system adapted from [8].

\subsection{Design of Experiments (DOE)}

The CCD is a technique of DOE, it was developed to analyze two or more factors that can have interaction between them. These factors can have some levels; levels work in an interval; edges of this interval are defined as high and low levels. However, graphic representation of the model is impossible when having more than three factors. Usually, only the three factors change in every iteration. The remaining factors are fixed in order to represent the model graphically.

Jang's [8, 19] presents a schematic representation of a model called Type-3 ANFIS (see Figure 2a). This particular case presents nine rules in their partition space, this model would be equivalent to (2), where the dimension of space (quantity of variables) $N$ represents the levels and $k$ represents the factors (Fuzzy sets) that need to be evaluated. In this case a $2^{\mathrm{k}}$ model (see Figure $2 \mathrm{~b}$ ) was selected, this model have maximum and minimum levels are known as treatments, also called axial points (see vertex of Figure 2b), this point was used as fuzzy sets that represents the inputs to the system the low and high levels represents the centers of the fuzzy sets to conform the rules. This model is very useful in the beginning of the experiments because it only needs a few tests to evaluate the behavior of the model in a confidence interval.

For the specific case of CCD, the model needs a series of samples with different combinations of the factors in order to accomplish the most possible states; this is mentioned in [24]. However, in this particular case was used to perform the rule reduction in the FLS and the ANFIS models with the use of the limits only. Commonly, all possible combinations of the levels are produced, in this case, four combinations (which are shown as sub-spaces in Figure 2a). 


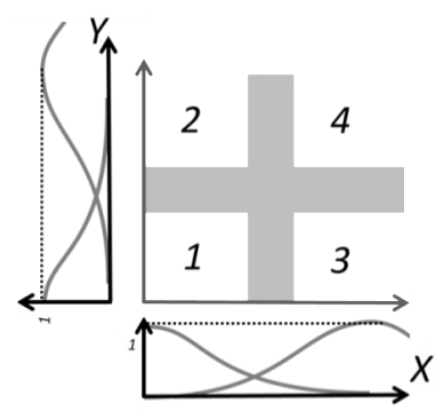

a)

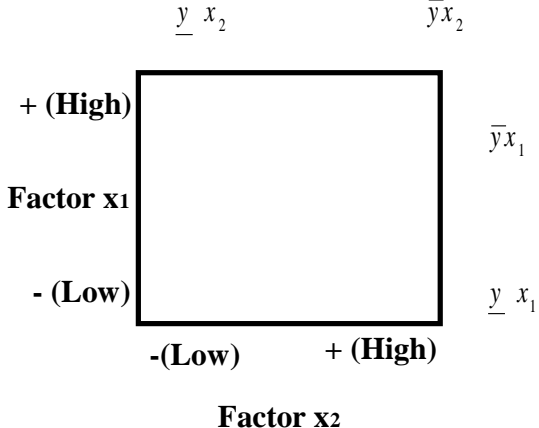

b)

Fig. 2. a) Type-3 ANFIS with four rules. Adapted from $[8,19]$. b) Factorial experiment $2^{k}$ with response (y) at the vertex. Adapted from [24].

$$
N^{k}
$$

Calculation of the principal effects of the axial points is needed to complete the model. Their values proceed by the combinations of the levels in every factor. In CCD the factors (for the fuzzy case variables $x_{1}$ and $x_{2}$ ) are called $\boldsymbol{A}$ and $\boldsymbol{B}$ in the case of high levels (for the lower levels $\boldsymbol{a}$ and $\boldsymbol{b}$ are used). The combinations are described with letters. The low levels are omitted in the symbolic representation. E.g. $\boldsymbol{a b}$ represents both variables in high level, $\boldsymbol{b}$ is used with the combination of $\boldsymbol{a}$ low and $\boldsymbol{b}$ high, $\boldsymbol{a}$ high and $\boldsymbol{b}$ low yields $\boldsymbol{a}$, finally $\boldsymbol{l}$ is used for both in low level (see Figure 3 ).

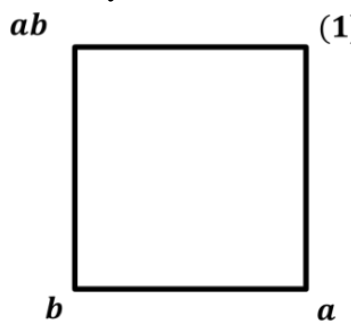

Fig. 3. CCD model graphical adaptation, adapted from [25].

The principal effect of $A$ is obtained by a combination of $n$ replies or samples and their effects in the low and high levels, namely the $A$ effect in the low level of $B$ is given by (3), in the other hand the $A$ effect in the high level of $B$ yields (4). Then the principal effect of $A$ is obtained by the combination of $(3,4)$ and yields $(5)$. A same procedure is used to obtain the principal effects of $B$, the $B$ effect on low level of $A$ is given by (6), the $B$ effect on the high level of $A$ yields (7) and the principal effect of $B$ is given by (8) finally the effect of $\mathrm{AB}$ is given by (9).

$$
\begin{gathered}
\bar{y}_{A^{-}=\frac{a-(1)}{2 n}} \\
\bar{y}_{A^{+}=\frac{a b-b}{2 n}} \\
A=\bar{y}_{A^{+}}-\bar{y}_{A^{-}}
\end{gathered}
$$




$$
\begin{gathered}
\bar{y}_{B^{-}=\frac{a+(1)}{2 n}} \\
\bar{y}_{B^{+}=\frac{a b+b}{2 n}} \\
B=\bar{y}_{B^{+}}-\bar{y}_{B^{-}} \\
A B=\bar{y}_{B^{+}} \bar{y}_{A^{-}}
\end{gathered}
$$

In order to complete the $\mathrm{CCD}$ to obtain the coefficients to produce the regression model based on the obtained principal effects of the treatments. The correlation coefficients are obtained by (10-12).

$$
\begin{gathered}
\beta_{0}=\bar{X}=\sum_{i=1}^{n} x_{i} \\
\beta_{1}=\sum_{i=1}^{n} a_{i} \\
\beta_{2}=\frac{\sum_{i=1}^{n} b_{i}}{\sum_{i=1}^{n} a_{i}}
\end{gathered}
$$

\subsection{Prediction Model}

The least squares (LS) method is used to predict the value of a function of time $t$ in [24]. The linear multivariate model can be expressed as a single equation (13). This is a matrix series that produces the pseudo-inverse matrix that results in the final function and then can be predicted a value at time $t$ (14). The obtained model is the same of the CCD and is used to predict some value at a point in time and evaluate their behavior.

$$
\begin{gathered}
\hat{\beta}=\left(X^{T} X\right)^{-1} X^{T} y \\
y=\beta_{0}+\beta_{1} x_{1}+\beta_{2} x_{2}+\cdots+\beta_{k} x_{k}+\varepsilon
\end{gathered}
$$

\section{Proposal}

This work evaluates the methodologies to model fuzzy systems in the pure and hybrid forms (FLS, FLS/CCD [25], ANFIS, ANFIS/CCD [12] and FLS IBISPC [26]) to confirm the accuracy of these methods. The reduced rule base used in it saves computational time without decrement the capacity of prediction of the model.

The design of these systems is based on the fact that there are no actual methodologies in literature or only exist a few methods to model this kind of expert systems (ES). Basically the design of the ES is based on the knowledge and experience of the designer and he decides how many sets need to be created for every variable.

The models mentioned above are created with the use of the natural separations of the models to divide the universe of discourse. E.g. CCD produces a square when has 2 variables (see Figure 4). Statistical process control (SPC) uses the Gaussian distribution and it has seven divisions then if (2) is applied the CCD has 4 rules in the ES and SPC has 49 rules. These rules are produced by mixed combinations of the graphical models for 2 variables also if the model has more than 3 variables are impossible to generate a graphical model then is needed fix some parameters in order 
to obtain a 3 variable model to see this graphically. The $2^{\mathrm{k}}$ factorial model gives the rule base for the ES (see Table 1). This is called principal effect or interaction matrix. It can represent a function that describes the model (15) as a multivariate function of first order.

$$
\text { a1x1@a2x2@...@anxn=y }
$$

Where $a$ is a scalar and @ is a mathematical operator.

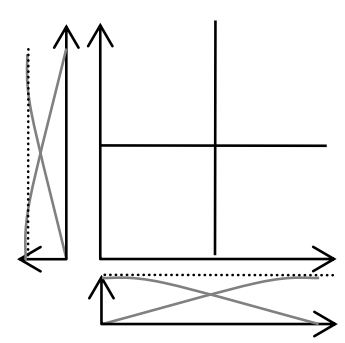

a)

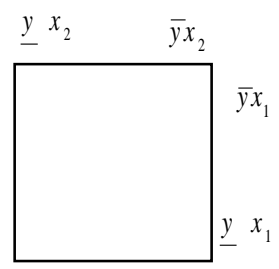

Factor $\mathrm{x} 2$

b)

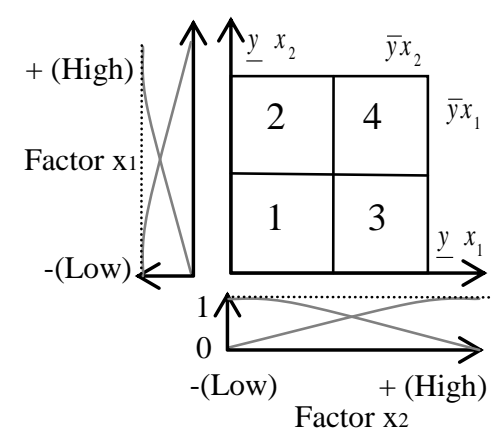

c)

Fig. 4. Symbolic adaptation of CCD model. Adapted from [25]. a) Jang's ANFIS model, b) $2^{k}$ Factorial design, c) Hybrid model.

Table 1 Values for inputs of CCD states of every variable and combinatorial treatment.

\begin{tabular}{ccccc}
\hline \multicolumn{2}{c}{ Variable } & \multicolumn{2}{c}{ Condition } & Treatment \\
\hline A & B & Low & Low & $\mathrm{A}_{\text {low }}, \mathrm{B}_{\text {low }}$ \\
A & B & High & Low & $\mathrm{A}_{\text {high }}, \mathrm{B}_{\text {low }}$ \\
A & B & Low & High & $\mathrm{A}_{\text {low }}, \mathrm{B}_{\text {High }}$ \\
A & B & High & High & $\mathrm{A}_{\text {high }}, \mathrm{B}_{\text {High }}$ \\
\hline
\end{tabular}

\section{Method}

The method used for modeling the ES is described in Figure 5. First, we need to use one of the described methodologies of individual base inference -such as IBISPC or 
CCD- to create the universe of discourse (UOD). The partition of UOD need to be modeled with the antecedents for later obtaining the consequents (if they exists, if not create the consequents by LS). Finally, run the ES to predict any state or combination of inputs for the system.

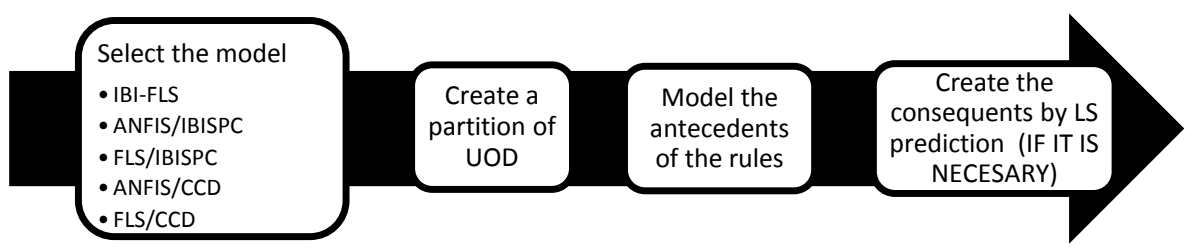

Fig. 5. Proposal for the methodologies comparison.

The next subsections describe the methods used to model-up the ES systems.

\subsection{Tests}

\section{Test A}

The IBI model uses as UOD of 11 sets for every variable and every data pair represents a rule. The final ES has 11 rules (see Table 2).

Table 2. Value for antecedents and consequents obtained by CCD (adapted from [9]).

\begin{tabular}{ccc}
\hline & Inputs & Output \\
X1, Tool feed, RPM & X2, Spindle speed, $\mathbf{M m} / \mathbf{m i n}$ & $\begin{array}{c}\text { Diameter }(\mathbf{m m}) \\
\text { Din }\end{array}$ \\
\hline 2500.00 & 300.000 & 4.18 \\
2750.00 & 275.000 & 4.08 \\
3000.00 & 250.000 & 4.19 \\
2750.00 & 239.645 & 4.07 \\
2396.45 & 275.000 & 4.15 \\
2750.00 & 310.355 & 4.10 \\
2750.00 & 275.000 & 4.12 \\
2500.00 & 250.000 & 4.17 \\
3103.55 & 275.000 & 4.08 \\
3000.00 & 300.000 & 3.97 \\
2750.00 & 275.000 & 4.03 \\
\hline
\end{tabular}

\section{Test B}

The IBISPC produces 7 sets for every variable and with (2), the missing consequents were obtained by prediction with LS, which uses the coefficients presented in Table 2 as their input, this represents the antecedents for the rules of FLS.

\section{Test C}

The CCD model produces only 2 sets per variable and their combination produces 4 rules for the system. Table 2 data was used to predict the output for the unknown 
states of the system without the need of more tests in the machining process that condition produces savings in resources because the missing states were predicted or simulated.

\section{Test D}

Evaluate the model (FLS, ANFIS, ANFIS/CCD or FLS/CCD). These models use the same UOD in both cases.

\section{Experimental Results}

Experiments confirm that the process works in the form of cycles such as a periodic function. The CCD model performs an evaluation to describe the states of the process as a control system. This state takes the minimum and maximum values of every variable and their permutations are called axial points in CCD. It can be transformed into fuzzy rules. Usually the number of fuzzy sets is calculated with the permutations of the variables as a thumb rule. It yields (16) and is a partition of UOD represented by the axial points in this case. The developed test runs 11 samples and 6 different numbers of epochs for every sample.

$$
F S^{x}
$$

Where $F S=\#$ of fuzzy sets and $X=\#$ variables

The rule reduction can be done with the study of performance of the process. This brings a periodic function behavior (see Figure 6). $Y$ axis represents the output for the ES: the function represents the system and its units are the diameter of the drilling operation. $X$ axis represents the different test (one was simulated and the remaining ones were actual physical tests). The frequency is given by the tool feed. The spindle speed explains the amplitude. The experiment can be made with a single period as a reduced base of rules. The results obtained by one period simplification in the form of DOE method also provide a novel model to perform a rule reduction.

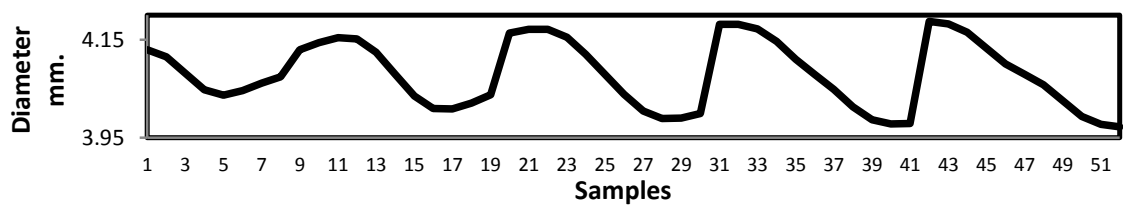

Fig. 6. Performance of machining process, response case (simulated).

The rule reduction is performed as following:

- Taking a period of the output

- As shown in Figure 3: the point in the left lower corner represents the lowest state of the system, the upper right corner represents the maximum state of the system as axial points for the spindle speed, the mean value represents the middle points for both axis. The crossover points are represented by intervals between the minimum or maximum and the mean. 
The rest of rules can be obtained by linear combinations of the axial points with the scalar product and these combinations are not necessary for the systems because the activation of these systems is always done at least with a pair of rules. Figure 7 shows a period in the response function, this function only found a change in the amplitude when the speed spindle changes (RPM). If the speed arises the amplitude arises and the frequency is constant at the time.

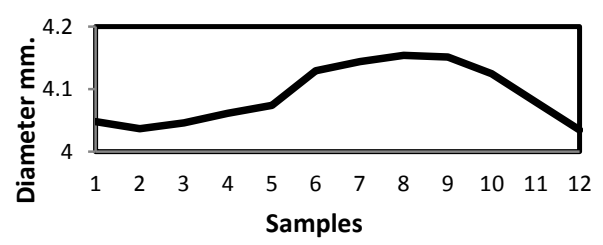

Fig. 7. Periodic performances for every fuzzy set of tool feed.

Figure 8 shows the performance of the systems. The chaotic behavior of the function precedes the nature of treatments of the CCD method. Tests need to be performed in a random order to avoid the appearance of either noise in the system or the cause of a trend. On the other hand, the models used (LS, FLS, ANFIS) are not exact methods and they can only make approximations of a function. Some variations of this performance can be explained by the wear and tear of the tool. Just as same, the similarity in the approximations generated by the purity or hybrid models and produced by the pre-processing of these methods is based in a fuzzification of the data and the variations in the processing of the following phases small variations due to differences in the calculated decimal by the nature of the model.

The sample number 10, of the table 2 is a special case because the variation can be caused by deterioration of the tool or can be considered as an atypical point. Anyway, this can be considered in a later study. Figure 8a shows the results obtained by the fuzzy systems (IBI and IBISPC) and hybrid form (FLSCCD). The differences between the error rate in FLS and optimized FLS are no significant in comparison of the savings in time and modeling (see Table 3 ). The results obtained by the hybrid ANFIS with the CCD show that this class of systems can be actually made. The error rate in ANFIS compared against the goal values is very similar to an FLS model (see Figure $8 \mathrm{~b}$ ). These models show good approximation capabilities. The computational time consumed in the CCD models is half in comparison of the IBI model and $10 \%$ in comparison of IBISPC model all results are shown in Table 4.

Table 3. Computational time of different tests with 10 epochs of training (seconds).

\begin{tabular}{cccccc}
\hline FLSCCD & FLSIBI & FLSISIBISPC & ANFISCCD & ANFISIBI & ANFISIBISPC \\
\hline $\mathbf{0 . 0 2 6}$ & 0.053 & 0.267 & $\mathbf{0 . 2 2 0}$ & 0.039 & 0.125 \\
\hline
\end{tabular}

Table 4. MSE of different models 10 epochs of training.

\begin{tabular}{cccccc}
\hline FLSCCD & FLSIBI & FLSISIBISPC & ANFISCCD & ANFISIBI & ANFISIBISPC \\
\hline $\mathbf{0 . 0 0 2 4}$ & $\mathbf{0 . 0 0 1 8}$ & 0.0030 & 0.0025 & 0.0031 & 0.0030 \\
\hline
\end{tabular}




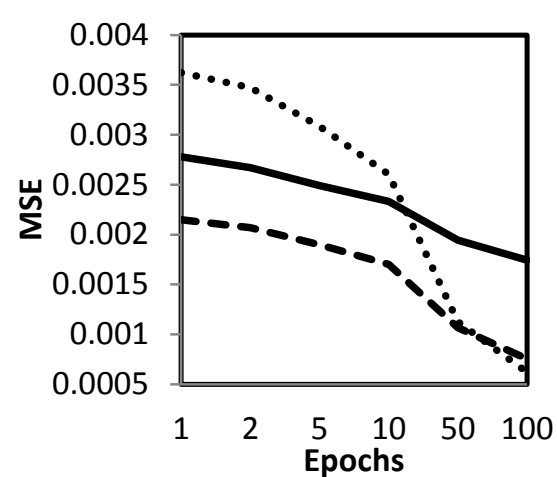

a)

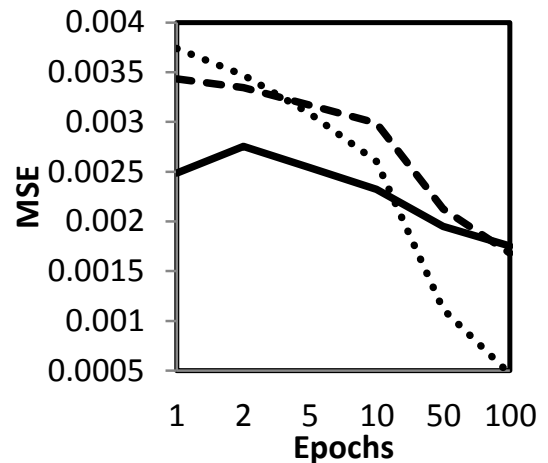

b)

Figure 8. MSE of different models. a) Fuzzy models, b) ANFIS models. Where: solid lines are CCD, dotted lines are IBISPC, and dashed lines are IBI.

\section{Conclusion}

The DOE techniques provide a simplification for modeling SC systems. The interrelation generated by the CCD serves as functions that can be used to model the rules in the form described in (1). These design techniques can also reduce the modeling work, because the analysis made by CCD evaluates the interaction between variables and with these interactions non-correlated variables can be eliminated of the model.

These kinds of systems were quite useful in generating an approximation or prediction to study the possible performance of the process in different conditions using less resources. Tests can be made by simulations with SC techniques. A complete extended rule base SC model with hybrid forms (FLS/CCD OR ANFIS/CCD) can prove its performance against a pure form (IBISPC) at least in this case.

The performance of the systems shows that the CCD can be used as a good model to calculate an approximation to FLS and ANFIS systems with a reduced rule base without having to worry about losing reliability. The use of these hybrid models (FLS/DOE and ANFIS/DOE) can reduce the size of the model around $80 \%$ without decrement the capacity of prediction (in comparison with the individual base inference model). Also, it can reduce the training cycles depending on the precision required.

\section{References}

1. Vale, Z., Morais, H., Faria, P., Ramos, C.: Distribution system operation supported by contextual energy resource management based on intelligent SCADA. Renewable Energy, vol. 52, pp. 143-153 (2013) 
2. Gutowski T.: Machining. Lectures of manufacturing processes and systems http://web.mit.edu/2.810/www/lecture09/4.pdf (2009)

3. Özel T., Davim P.: Intelligent Machining, control systems, robotics and manufacturing series. 1st edition. Wiley-ISTE. pp. 1-61 (2009)

4. Krishnamoorthy, A., Rajendra Boopathy, S., Palanikumar, K., Paulo Davim, J.: Application of grey fuzzy logic for the optimization of drilling parameters for CFRP composites with multiple performance characteristics. Measurement, 45(5), pp. 1286-1296 (2012)

5. Fujipress.: CALL FOR PAPERS, Special Issue on Machining of Difficult-to-cut Materials for the International Journal of Automation Technology (IJAT), Vol.7 No.3 (2013)

6. Haber, R. E., del Toro, R. M., Gajate, A.: Optimal fuzzy control system using the crossentropy method. A case study of a drilling process. Information Sciences, 180(14), pp. 2777-2792 (2010)

7. Rajmohan, T., Palanikumar, K., Prakash, S.: Grey-fuzzy algorithm to optimise machining parameters in drilling of hybrid metal matrix composites. Composites Part B: Engineering, 50, pp. 297-308 (2013)

8. Jang, J. S.: ANFIS: adaptive-network-based fuzzy inference system. Systems, Man and Cybernetics, IEEE Transactions on, 23(3), pp. 665-685 (1993)

9. Kabini, K.: Review of ANFIS and its Application in Control of Machining Processes. Sustainable Research and Innovation Proceedings (2011)

10. Praga-Alejo R., González G. D., Pérez V. P, Cantú S. M., Flores H. B.: Modeling a Fuzzy Logic System Using Central Composite Design. Proceedings of 1 st annual world Conference of the Society for Industrial and Systems Engineering. Washington D.C., USA, September 16-18 (2012)

11. Makadia, A. J., Nanavati, J. I.: Optimisation of machining parameters for turning operations based on response surface methodology. Measurement, 46(4), pp. 1521-1529 (2013)

12. Montes Dorantes P. N., Praga-Alejo R., Nieto Gonzalez J.P., Méndez G.M.: Modelado de Sistemas Adaptativos de Inferencia Neuro-Difusa Usando Diseño Central Compuesto. Research In Computing Science, vol. 62, pp. 259-269 (2013)

13. Borrotti M., Poli I.: Naïve Bayes Ant Colony Optimization for Experimental Design. Synergies of Soft Computing and Statistics for Intelligent Data Analysis Advances in Intelligent Systems and Computing Volume 190, 2013, pp 489-497, Springer-Verlag (2013)

14. Balasko B., Madar J., Abonyi J.: Additive Sequential Evolutionary Design of Experiments. Artificial Intelligence and Soft Computing - ICAISC 2006. Lecture Notes in Computer Science Volume 4029, 2006, pp 324-333. Springer-Verlag (2006)

15. Yu J., Tsung R.H, Juang J., Thibault F.: Design Optimization Using Soft Computing Techniques for Extrusion Blow Molding Processes. 2002 NRC-NSC Canada-Taiwan Joint Workshop on Advanced Manufacturing Technologies, London, Canada. pp. 73-84 (2002)

16. Zadeh L.A.: Fuzzy Sets. Information and control 8(3), pp.338-353 (1965)

17. Zadeh L.A.: The concept of a linguistic variable and its application to approximate reasoning I. Information Sciences, 8(1975). pp.199-249 (1975)

18. Mamdani, E. H., Assilian, S.: An experiment in linguistic synthesis with a fuzzy logic controller. International journal of man-machine studies, 7(1), pp. 1-13 (1975)

19. Jang, J. S. R., Sun, C. T.: Neuro-fuzzy and soft computing: a computational approach to learning and machine intelligence. Prentice-Hall, Inc. (1996)

20. Hosseini, M. S., Zekri, M.: Review of Medical Image Classification using the Adaptive Neuro-Fuzzy Inference System. Journal of medical signals and sensors, 2(1), 49 (2012)

21. Mendez, G. M., de los Angeles Hernandez, M.: IT2 TSK NSFLS2 ANFIS. In Artificial Intelligence (MICAI), 2010 Ninth Mexican International Conference on. pp. 89-93, IEEE, (2010) 
22. González M. A., \& Ballarin V. L.: Segmentación de imágenes utilizando la transformada Watershed: obtención de marcadores mediante lógica difusa IEEE latin america transactions, 6(2), pp. 223-228 (2008)

23. Dutra R. G., \& Martucci Jr. M.: Adaptive Fuzzy Neural Tree Network IEEE latin america transactions, 6(5).pp. 456-460 (2008)

24. Montgomery D.C: Diseño y Análisis de experimentos. $2^{\text {a }}$ Ed. Limusa-Wiley. pp.218 - 279 (2004)

25. Montes Dorantes P. N., Nieto González J.P., Praga-Alejo R., Guajardo Cosio K.L., \& Méndez G.M.: Sistema inteligente para procesamiento de imágenes en control de calidad basado en el modelo difuso singleton tipo 1. RCS 74, pp. 117-130 (2014)

26. Montes Dorantes P. N., Nieto González J.P., Praga-Alejo R., \& Méndez G.M.: Fault Detection Systems via a Novel Hybrid Methodology for Fuzzy Logic Systems Based on Individual Base Inference and Statistical Process Control. IEEE latin america transactions, 12(4), pp. 706-712 (2014) 